\title{
Hepatic Artery Pseudoaneurysm with Extrahepatic Biliary Obstruction
}

\author{
Manabu Shiraishi, MD, Masaya Takahashi, MD, PhD, Atsushi Yamaguchi, MD, PhD, and \\ Hideo Adachi, MD, PhD
}

Hepatic artery pseudoaneurysms are a rare complication of chronic pancreatitis. However, giant pseudoaneurysms $(80 \mathrm{~mm})$ and complicated biliary obstructions are extremely rare. This article reports a 75 year-old man with chronic pancreatitis that presented as upper abdominal pain and obstructive jaundice. Computed tomography revealed a hepatic artery pseudoaneurysm measuring $80 \times 72 \times 70 \mathrm{~mm}$. We performed aneurysm resection and common hepatic artery bypass, using a great saphenous vein graft in the patient. The postoperative course was uneventful, and he was discharged on the 10th postoperative day. However, on the 135th postoperative day, he died of massive hemorrhage into the peritoneal cavity.

Keywords: visceral artery, hepatic artery, pseudoaneurysm

\section{INTRODUCTION}

$\mathrm{V}$ isceral artery aneurysms are rare but potentially life-threatening. Pseudoaneurysms that occur as a consequence of chronic pancreatitis are associated with significant morbidity and mortality because of potential bleeding. Major hemorrhage is a rare but lethal complication of pancreatitis. Rupture may present as either fatal bleeding into a pseudocyst, the peritoneal cavity, or the retroperitoneum, or erosion into the stomach, pancreatic duct, or bile ducts. ${ }^{1,2}$ Pseudoaneurysm in conjunction with chronic pancreatitis often occurs in association with the splenic artery. Hepatic artery aneurysms are comparatively rare. Traditional therapy has been surgical resection or ligation or endovascular embolization. These treatment options depend on the presentation of the patient and location of the aneurysm. ${ }^{3)}$ We herein describe a case of a giant pseudoaneurysm of the common hepatic artery in a patient who presented with abdominal pain

Division of Cardiovascular Surgery, Saitama Medical Center, Jichi Medical University, Saitama, Saitama, Japan

Received: October 27, 2011; Accepted: December 25, 2011 Corresponding author: Manabu Shiraishi, MD. Division of Cardiovascular Surgery, Saitama Medical Center, Jichi Medical University, 1-847 Amanuma, Omiya-Ku, Saitama, Saitama 330-8503, Japan Tel: +81-48-647-2111, Fax: +81-48-648-5188

E-mail:manabu@omiya.jichi.ac.jp and extrahepatic biliary obstruction without bleeding.

\section{Case Report}

The patient was a 75 year-old man who was an alcoholic and former smoker. He had a 2 year history of longstanding epigastric pain. Chronic pancreatitis had been previously detected and was controlled. There was no history of open surgery. Abdominal examination results showed a pulsating palpable mass, and blood examination results revealed significantly elevated serum total bilirubin (1.89 mg/dl), serum direct bilirubin (1.27 mg/dl), aspartate aminotransferase $(305 \mathrm{mU} / \mathrm{ml})$, alanine aminotransferase $(207 \mathrm{mU} / \mathrm{ml})$, alkaline phosphatase $(693 \mathrm{mU} / \mathrm{ml})$, and gamma-guanosine triphosphate $(291 \mathrm{mU} / \mathrm{ml})$. He was subsequently diagnosed with arterial aneurysm and obstructive jaundice without symptoms of icterus. The patient was admitted to our center and underwent further evaluation.

The patient was initially evaluated elsewhere with abdominal ultrasonography, which revealed distention of the intrahepatic bile duct, stenosis of the lower part of the common bile duct, and an aneurysm $(80 \times 72 \times$ $70 \mathrm{~mm}$ ) contiguous with the celiac artery. We performed computed tomography (CT) with intravenous contrast to assess the nature of the mass. The scan revealed a heterogeneous $80 \times 72 \times 70-\mathrm{mm}$ mass involving the 
head of the pancreas (Fig. 1a) and features of chronic pancreatitis with multiple foci of calcifications in the pancreatic duct and parenchyma involving the body and tail. The pancreatic duct was dilated. However, the stage of chronic pancreatitis was not clear, the inflammation of the pancreas had not been active, and exacerbation of the pancreatitis was not detected in CT. Three-dimensional CT (3D-CT) revealed that the aneurysm arose from the origin of the common hepatic artery (Fig. 1b). Selective arteriography of the celiac trunk revealed the irregular lumen of the common hepatic artery filled with an intramural thrombus (Fig. 1c). The proper hepatic artery and right gastric artery arose from the pseudoaneurysm. The bifurcation of the gastroduodenal artery was not clearly detected on preoperative angiography or CT. The pseudoaneurysm was caused by rupture of the common hepatic arterial wall. Any other aneurysm was not detected on the preoperative examinations. Because there were no signs of rupture or bleeding, the patient was a candidate for elective treatment. The patient fasted and did not undergo preoperative reduction for cholestasis. The blood chemistry values were moderately improved. In addition to the large size of the pseudoaneurysm, which placed it at risk for rupture, and the difficult location for arterial embolization, the patient had no risk factors for surgery. Therefore, it was determined that he would be treated surgically.

The operation was performed through an upper median laparotomy. The pseudoaneurysm was densely adhered to the stomach and retroperitoneum. The lesser sac was entered by dividing the gastrocolic omentum. The stomach was separated from the pseudoaneurysm and pancreas by careful dissection (Fig. 2). Proximal vascular control of the common hepatic artery was achieved near its origin. Because the outflow artery from the aneurysm was densely adhered to the aneurysmal wall, we closed the outflow (right gastric artery and proper hepatic artery) from the inside of the aneurysm. The inflow and outflow vessels were ligated, and the aneurysm was resected. A distal and proximal common hepatic artery bypass using a great saphenous vein graft was then performed.

The postoperative period was uneventful, and followup CT on the 8th postoperative day revealed successful anastomosis of the common hepatic artery and vein graft (Fig. 3). The blood chemistry values were promptly improved to normal levels. The patient was discharged on the 10th postoperative day. He visited the hospital for treatment once during several months without complications. However, on the 135 th postoperative day, he was

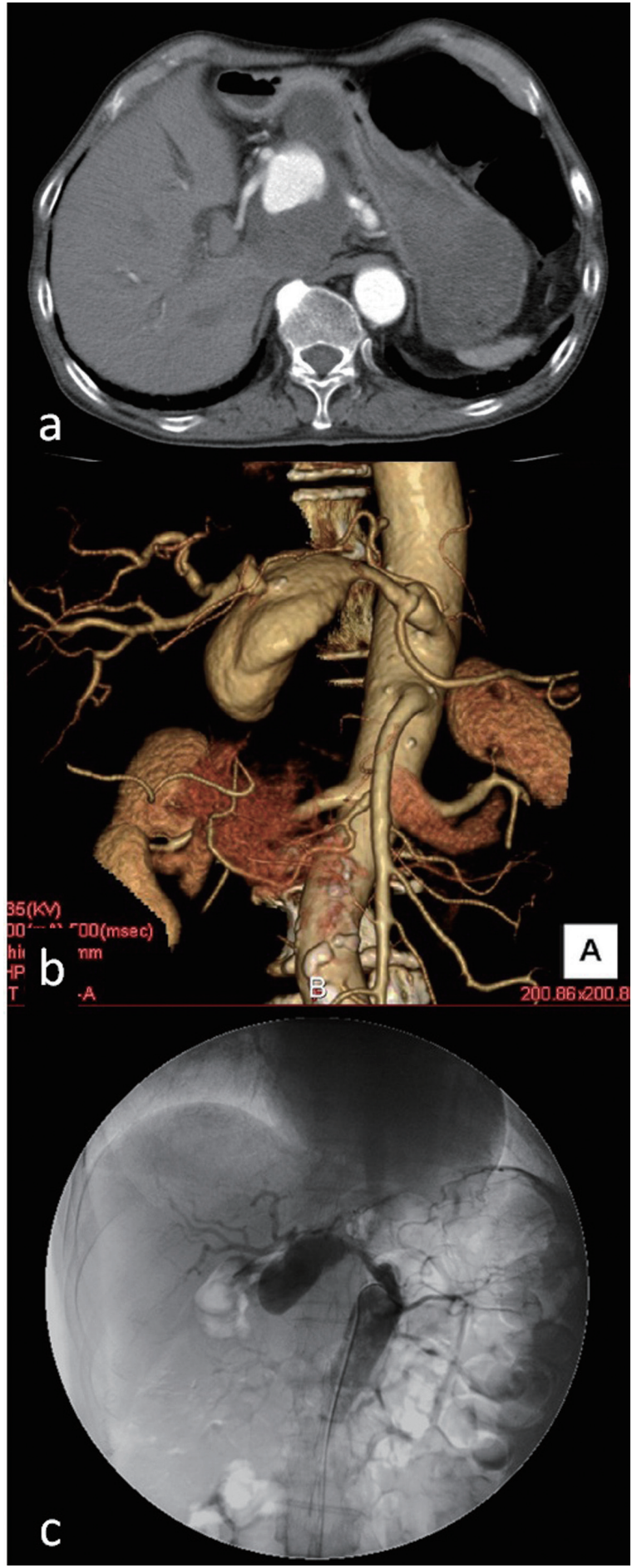

Fig. 1 (a) Abdominal contrast-enhanced computed tomography showed a pseudoaneurysm measuring $80 \times 72 \mathrm{~mm}$ on the ventral side of the pancreatic head. (b) Three-dimensional computed tomography showed that the aneurysm arose from a branch of the common hepatic artery. (c) A celiac axis angiogram confirmed that the pseudoaneurysm arose from the common hepatic artery and showed that the irregular lumen of the common hepatic artery was filled with the intramural thrombus. 


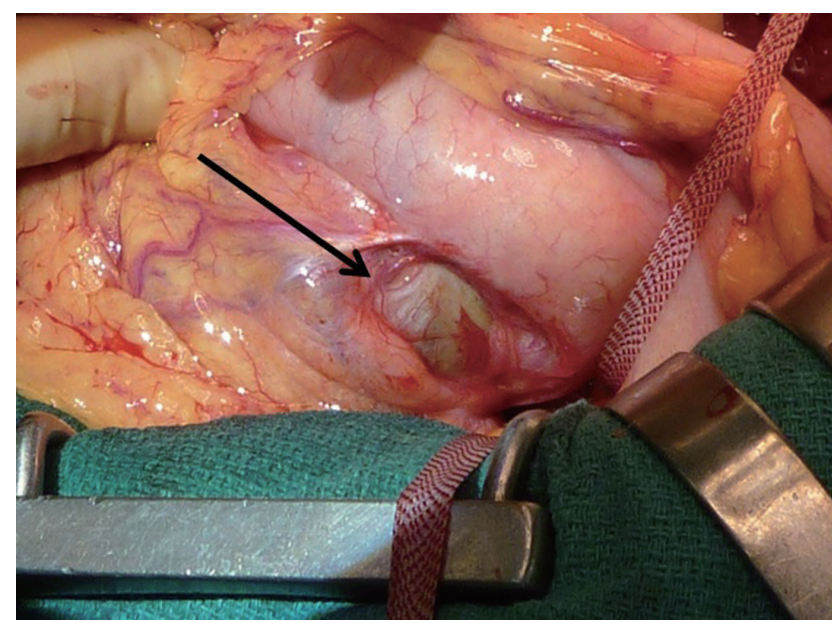

Fig. 2 Intraoperative photograph showed the hepatic artery pseudoaneurysm. Solid arrow indicates the pseudoaneurysm.

transported to our center with cardiopulmonary arrest and was confirmed dead. From the autopsy, the cause of death was assumed to be massive hemorrhage into the peritoneal cavity from the artery around the head of the pancreas. Any other aneurysm was not newly detected. There were no problems associated with the common hepatic artery bypass site.

\section{Discussion}

Visceral artery pseudoaneurysms are uncommon. They usually develop secondary to pancreatic disease, with incidence ranging between $10 \%$ and $17 \%$ in patients with chronic pancreatitis. They have been reported as a late complication, occurring an average of 2 to 3 years after the first episode of pancreatitis, after repeated acute bouts in patients with chronic pancreatitis. ${ }^{4,5)}$ The splenic artery is the most commonly involved vessel, accounting for half of cases of pseudoaneurysms in chronic pancreatitis, particularly because of its anatomic location. ${ }^{6}$ The frequency of hepatic artery pseudoaneurysms due to chronic pancreatitis is comparatively uncommon at $2 \%$ to $12 \% .{ }^{2,7)}$ However, since the $1980 \mathrm{~s}$, the growing use of percutaneous diagnostic and endoscope therapeutic procedures on the biliary tract has resulted in an increased number of visceral artery pseudoaneurysms, especially of the hepatic artery. ${ }^{7)}$

Rupture of a pseudoaneurysm in the gastrointestinal tract is a life-threatening event that is associated with

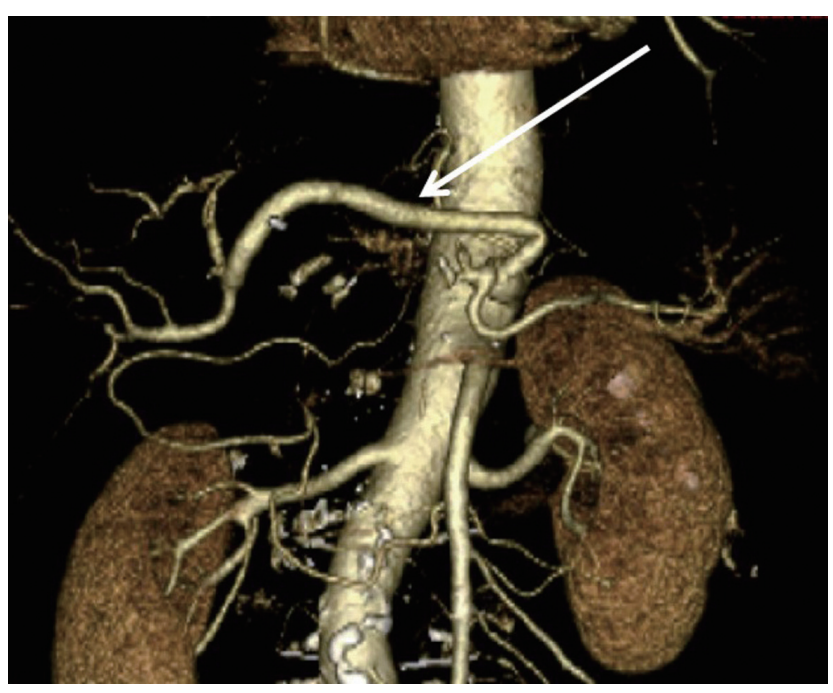

Fig. 3 Follow-up 3-dimensional computed tomography showed successful anastomosis. Solid arrow indicates the saphenous vein graft.

a mortality rate of approximately $75 \%$ to $90 \% .^{2,6)}$ The pathogenesis of hepatic artery pseudoaneurysms is not well understood. Pancreatitis, abdominal trauma, and iatrogenic causes (e.g., prior abdominal surgery or endovascular interventions) have been reported as risk factors for hepatic artery pseudoaneurysm. ${ }^{1,3,6,8)}$ In the present case, chronic pancreatitis seemed to play a role in weakening the common hepatic artery wall. The mechanisms of pseudoaneurysm formation in pancreatitis include inflammation with enzymatic digestion of the pancreatic or peripancreatic artery, visceral artery erosion by pseudocysts, and pseudocyst erosion into the bowel wall and intramural artery. ${ }^{9)}$

Most pseudoaneurysms commonly present with abdominal pain or gastrointestinal hemorrhage or as a pulsatile abdominal mass accompanied by bruit. ${ }^{3,8)}$ As in this case, obstructive jaundice is an extremely rare clinical symptom of hepatic artery pseudoaneurysm.

Diagnosis is usually made from CT or transcatheter angiography results. Moreover, 3D-CT has been performed recently and is reportedly very useful for diagnosis, showing images of optimal directions and being less invasive. ${ }^{10)}$ In the present case, the location of pseudoaneurysm was easily diagnosed by $3 \mathrm{D}-\mathrm{CT}$. It revealed that the pseudoaneurysm arose from the common hepatic artery, and this was confirmed at surgery.

If a hepatic artery pseudoaneurysm ruptures, the patient faces a severely life-threatening condition that requires emergency treatment. However, because of its 
rarity, no strong consensus exists on the indications for treating visceral artery aneurysm. Marone et al. reported that $>2-\mathrm{cm}$ aneurysms with a rapid growth rate and symptoms attributable to aneurysm seem to be associated with conditions imposing aggressive treatment.7) The traditional therapy for visceral artery aneurysms has been surgical resection or ligation of the aneurysm. Recently, endovascular treatment (coil embolization and stent grafting) has been an alternative to open surgical repair and has become increasingly popular. ${ }^{4)}$ Because of the development of imaging techniques and percutaneous procedures, there are many reports of successful endovascular treatment and low morbidity and mortality. ${ }^{1,2,6-8)}$ On the other hand, there is a large study describing the management of hepatic artery aneurysm. It showed that endovascular treatment carries mortality and success rates similar to those of operative strategies. ${ }^{7)}$ The most frequent complication is the persistent blood flow in the pseudoaneurysm, which is usually caused by a proximal occlusion without distal closure of the artery, resulting in retrograde filling of the pseudoaneurysm through collateral vessels. ${ }^{6}$ ) It is difficult to completely pack a large saccular pseudoaneurysm with coils, leading to persistent expansion of the residual sac. Although an endovascular approach could represent a valid alternative for high-risk patients, surgical treatment remains the standard. ${ }^{7}$ Open surgery was the preferred approach in our case because of difficulty in embolization of the cavity, in addition to the need for surgical release of the common bile duct obstruction, by the pseudoaneurysm. However, occlusion of the common hepatic artery has no clinical consequences in the presence of a normally patent portal vein. Aneurysmectomy and bypass grafting were performed in our patient because the portal vein may have been affected by the compression of the aneurysmal wall and adhesion of postoperative inflammation.

In the late stage, one of the most important factors to consider in follow-up of patients is a recurrence of visceral aneurysms. Recurrence of hemorrhage from a pseudoaneurysm secondary to pancreatic inflammation has been reported. ${ }^{2)}$ In the present case, re-bleeding from arteries around the pancreas seemed to be the cause of death. Rebleeding from known pseudoaneurysms and development of further vascular abnormalities can occur, mandating careful clinical follow-up and repeat angiography as necessary.

\section{Conclusion}

Hepatic artery pseudoaneurysms with biliary obstruc- tion are extremely rare. Pseudoaneurysms are unrelated to the severity of pancreatitis, and major hemorrhage can occur irrespective of their size. Therefore, aggressive treatment should be considered once the diagnosis has been made. Endovascular treatment is safe and feasible in selected patients but is not suitable for large aneurysms with several collaterals. Treatment options should be selected based on the presentation of the patient and nature of the aneurysm. Regardless of the activity of the pancreatitis, visceral pseudoaneurysm may develop. We also believe that periodic abdominal imaging (e.g., abdominal ultrasonography or abdominal CT) should be performed to detect recurrence early.

\section{Disclosure Statement}

No external funding was obtained for the work herein presented.

\section{REFERENCES}

1) Skipworth J, Raptis D, Brennand D, et al. The management of multi-site, bleeding, visceral artery pseudoaneurysms, secondary to necrotising pancreatitis. Ann R Coll Surg Engl 2009; 91: 255-8. [Medline] [CrossRef]

2) Sethi H, Peddu P, Prachalias A, et al. Selective embolization for bleeding visceral artery pseudoaneurysms in patients with pancreatitis. Hepatobiliary Pancreat Dis Int 2010; 9: 634-8. [Medline]

3) Matsuno Y, Mori Y, Umeda Y, et al. Surgical repair of true gastroduodenal artery aneurysm: a case report. Vasc Endovascular Surg 2008; 42: 497-9. [Medline] [CrossRef]

4) de Perrot M, Berney T, Bühler L, et al. Management of bleeding pseudoaneurysms in patients with pancreatitis. Br J Surg 1999; 86: 29-32. [Medline] [CrossRef]

5) Balthazar EJ, Fisher LA. Hemorrhagic complications of pancreatitis: radiologic evaluation with emphasis on CT imaging. Pancreatology 2001; 1: 306-13. [Medline] [CrossRef]

6) Volpi MA, Voliovici E, Pinato F, et al. Pseudoaneurysm of the gastroduodenal artery secondary to chronic pancreatitis. Ann Vasc Surg 2010; 24: 1136. e7-11.

7) Marone EM, Mascia D, Kahlberg A, et al. Is open repair still the gold standard in visceral artery aneurysm management? Ann Vasc Surg 2011; 25: 936-46. [Medline] [CrossRef]

8) Gupta V, Kumar S, Kumar P, et al. Giant pseudoaneurysm of the splenic artery. JOP 2011; 12: 190-3. [Medline]

9) Hsu JT, Yeh CN, Hung CF, et al. Management and outcome of bleeding pseudoaneurysm associated with chronic pancreatitis. BMC Gastroenterol 2006; 6: 3 . [Medline] [CrossRef]

10) Matsuzaki Y, Inoue T, Kuwajima K, et al. Aneurysm of the gastroduodenal artery. Intern Med 1998; 37: 930-3. [Medline] [CrossRef] 